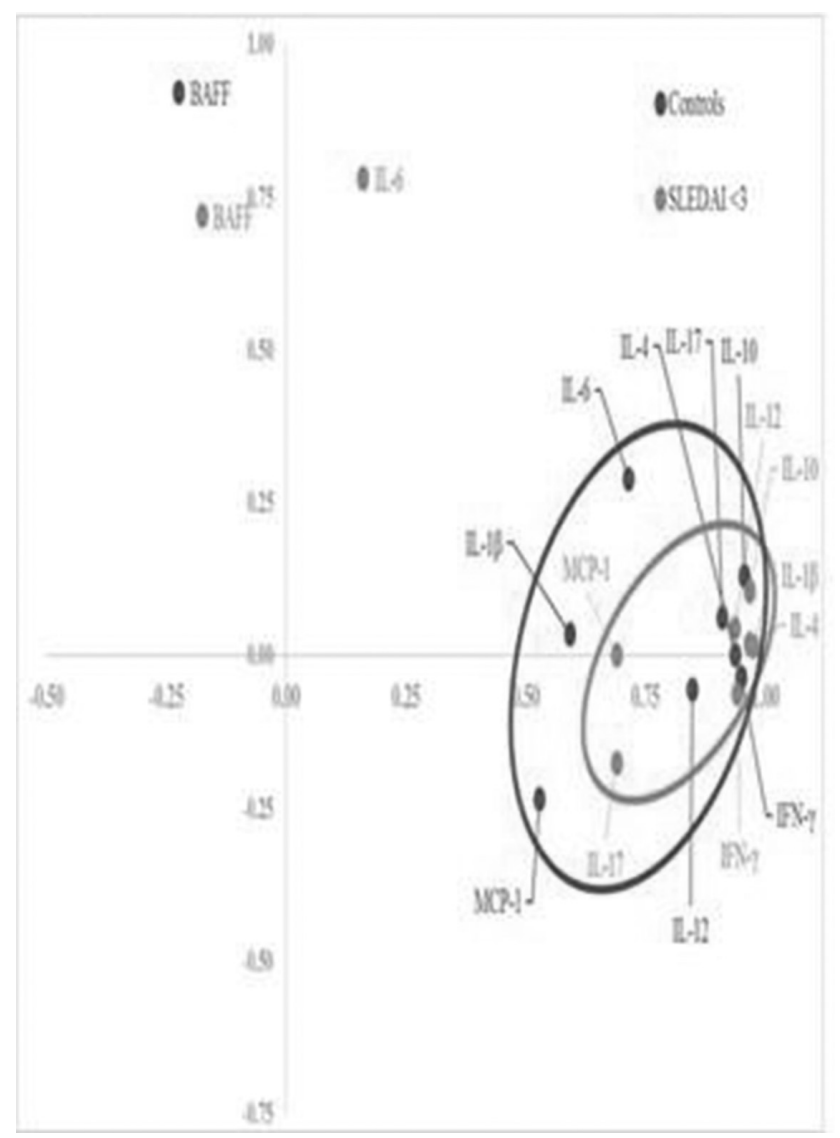

Abstract 418 Figure 2 Principal Component Analysis of Controls vs SLEDAl $<3$ group.

Conclusions Hospitalisation rates for SLE in WA have not decreased over 25 years. Once hospital-based management for SLE was required, the risk of all-cause mortality doubled compared to age and gender-matched controls. This risk was greatest in Medicare reliant or male SLE patients and not due to increased cancer risk.

\section{HOSPITALISATION FOR COMORBID CONDITIONS IN PATIENTS WITH SYSTEMIC LUPUS ERYTHEMATOSUS IS MORE FREQUENTLY DUE TO CARDIOVASCULAR AND RENAL COMPLICATIONS, SUBSEQUENTLY INCREASING THE RISK OF DEATH}

${ }^{1}$ W Raymond*, ${ }^{2} \mathrm{D}$ Preen, ${ }^{3} \mathrm{C}$ Inderjeeth, ${ }^{4} \mathrm{H}$ Keen, ${ }^{1} \mathrm{~J}$ Nossent. ${ }^{1}$ The University of Western Australia, Rheumatology Group - School of Medicine, Perth, Australia; ${ }^{2}$ The University of Western Australia, School of Population Health, Perth, Australia; ${ }^{3}$ Sir Charles Gairdner and Osborne Park Hospital Group, Rheumatology, Perth, Australia; ${ }^{4}$ Fiona Stanley Hospital, Rheumatology, Perth, Australia

\subsection{6/lupus-2017-000215.420}

Background and aims Systemic Lupus Erythematosus (SLE) is a chronic autoimmune disease that runs an unpredictable disease course. We aimed to understand the characteristics and outcomes of incident hospitalisation for conditions other than the underlying disease in SLE patients.

Methods Using whole-population data linkage of hospital admissions and death records in Western Australia (WA) WA

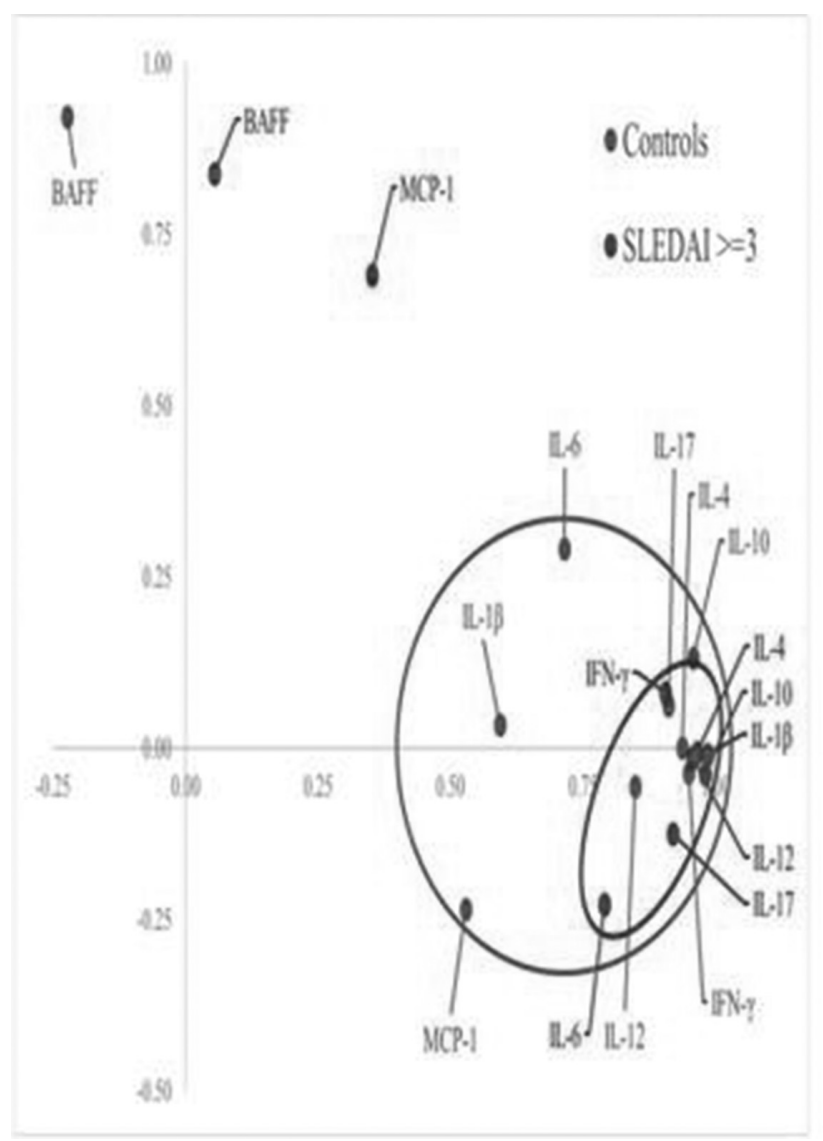

Abstract 418 Figure 3 Principal Component Analysis of Controls vs $\mathrm{SLEDAI} \geq 3$ group.

between 1980 and 2015, we performed a retrospective analysis for patients where SLE (ICD-9-CM 695.4, 710.0 and ICD10-AM L93.0 and M32) was a co-existing discharge diagnosis. All SLE patients were age- and gender-matched with hospital controls free of rheumatic disease. We investigated the rate and characteristics of the index hospitalisation for comorbidity and the risk of subsequent death by Kaplan-Meier survival and Cox regression.

Results Hospitalisation rates for comorbidity were 13.9/million/ year in SLE patients. SLE patients were similar to controls for age and gender, but more likely to be Indigenous, have renal failure, cardiovascular and thrombotic conditions (Table 1). Independent predictors of mortality risk following hospitalisation for a comorbid condition included: SLE diagnosis (OR 1.6, CI: 1.3-1.9, p<0.001) (Figure 1), cerebrovascular events (OR 2.0, CI: 1.2-3.7, p<0.001), renal disease (OR 1.75, CI: 1.4-2.3 p<0.001), thrombotic events (OR 1.8 CI: 1.1-2.8, $\mathrm{p}=0.001$ ) and reliance on Medicare (OR 1.5, CI: 1.3-1.8, $\mathrm{p}<0.001$ ) (Table 2).

Conclusions SLE patients were more frequently hospitalised than controls for cardiovascular or renal conditions and this increased their mortality risk. These results strengthen the need for close monitoring and interventions to prevent such comorbidity in all SLE patients. 